\title{
Pressure-induced transformations in two-dimensional polymeric phases of $\mathbf{C}_{60}$
}

\author{
(C) K.P. Meletov*,**, J. Arvanitidis*, S. Assimopoulos*, G.A. Kourouklis* \\ * Physics Division, School of Technology, Aristotle University of Thessaloniki, \\ GR-540 06 Thessaloniki, Greece \\ ${ }^{* *}$ Institute of Solid State Physics, Russian Academy of Sciences, \\ 142432 Chernogolovka, Moscow region, Russia
}

E-mail: mele@issp.ac.ru

\begin{abstract}
The structural stability of the tetragonal and rhombohedral two-dimensional (2D) polimeric phases of $\mathrm{C}_{60}$ has been studied under pressure up to $27 \mathrm{GPa}$ and room temperature by means of in-situ Raman scattering spectroscopy. The results show that the tetragonal 2D phase undergoes an irreversible transformation in the region of $20 \mathrm{GPa}$ while no pressure-induced transitions were observed for the rhombohedral 2D phase. The obtained data are discussed within the framework of recent numerical calculations, which predict the pressure-induced transformation of the 2D polymeric phases of $\mathrm{C}_{60}$ into three-dimensional (3D) polymers in the pressure range 14-20 GPa.
\end{abstract}

This work was supported, in part, by the Russian Foundation for Fundamental Research (grant N 99-02-17555). The support by the General Secretariat for Research and Technology (GSRT), Greece (grant N IENE $\Delta 99$, $99 \mathrm{E} \Delta / 62)$ is acnowledged.

The pristine $\mathrm{C}_{60}$ in the condensed phase has great potential for polymerization due to existence of 30 double $\mathrm{C}=\mathrm{C}$ bonds in the fullerene molecular cage. The covalent polymeric bonds, between adjacent molecules, are usually built up by the $[2+2]$ cyclo-addition mechanism, which results in the $s p^{3}$-like four-fold coordination of a number of carbon atoms of the molecular cage [1]. The crystal structures of the known polymeric phases of $\mathrm{C}_{60}$ were identified as one-dimensional (1D) orthorhombic, 2D tetragonal, 2D rhombohedral and 3D cubic [2-4]. These phases, as well as the so-called hard fullerite phases which are of 3D polymeric nature [5], can be prepared under various conditions of high pressure and high temperature treatment. Recent theoretical calculations have predicted that the $2 \mathrm{D}$ polymeric phase of $\mathrm{C}_{60}$ can be transformed, at room temperature, by uniaxial compression to a $3 \mathrm{D}$ polymaric phase [6]. This polymerization will take place at $P \approx 20 \mathrm{GPa}$ and results in the formation of a stable metallic phase having $24 s p^{3}$ - and $36 s p^{2}$-like coordinated carbon atoms in each $\mathrm{C}_{60}$ molecule. Another theoretical study [7] predicts that uniaxial compression up to $\sim 14 \mathrm{GPa}$ perpendicularly to the chains in $1 \mathrm{D}$ or planes in $2 \mathrm{D}$ polymers of $\mathrm{C}_{60}$ leads to the $3 \mathrm{D}$ polymerization with 52, 56 and even $60 s p^{3}$-like coordinated carbon atoms per molecule. To explore these predictions we have studied the pressure response of the 2D tetragonal and rhombohedral polymeric phases of $\mathrm{C}_{60}$ at pressures up to $\sim 27$ GPA by means of in-situ Raman scattering using the diamond anvil cell (DAC) technique.

\section{Experimental}

The 2D polymeric phases of $\mathrm{C}_{60}$ were obtained by high pressure and high temperature treatment of the $99.99 \%$ pure pristine $\mathrm{C}_{60}$ at $T=820 \mathrm{~K} P \approx 2.5 \mathrm{GPa}$ and $T=773 \mathrm{~K}$, $P \approx 5 \mathrm{GPa}$ for the tetragonal and rhombohedral polymers, respectively. Raman spactra were recorded using a triple monochromator (DILOR XY-500) equipped with a CCD liquid-nitrogen-cooled detector system. The $514.5 \mathrm{~nm}$ line of an $\mathrm{Ar}^{+}$laser with beam power of $2-10 \mathrm{~mW}$, measured befor the cell, was used for excitation. Measurements of the Raman spectra at high pressure were carried out using a DAC of the Mao-Bell type. The 4:1 methanolethanol mixture was used as pressure transmitting medium and the ruby fluorescence technique was used for pressure calibration. The specimens had dimensions of $\sim 100 \mu \mathrm{m}$ and have been selected from the batch material checking their Raman spectrum to be spatially uniform and typical of the corresponding phase.

\section{Results and discussion}

The Raman spectra of the 2D tetragonal polymer of $\mathrm{C}_{60}$ pressures up to $\sim 27 \mathrm{GPa}$ and room temperature are illustrated in Fig. 1. The initial spectrum (Fig. 1,a), taken at normal conditions, is identical with the spectra of this material reported earlier [8]. The spectrum is richer than that of the pristine $\mathrm{C}_{60}$ due to the lower symmetry of the molecule, which, in turn, results in the splitting of degenerate phonon modes and/or the activation of silent modes. As the pressure increases the Raman peaks shift to higher energies and their bandwidth gradually increases (Fig. 1, $b$, $P \approx 14.1 \mathrm{GPa})$. Dramatic changes are first recorded at the pressure of $\sim 20.7 \mathrm{GPa}$ (Fig. 1,c). New features appear which grow rapidly in intensity with increasing pressure up to the highest pressure attained in this study (Fig. 1, $d$, $P \approx 27.5 \mathrm{GPa}$ ). The transformation of the Raman spectrum is associated with the appearance of new intense peaks near $\sim 1000$ and $\sim 1920 \mathrm{~cm}^{-1}$ as well as the disappearance of some of the old ones. The total number of Raman active modes in the new spectrum is smaller whereas the intensity and position of other peaks differ considerably from those in the initial spectrum. The majority of the 


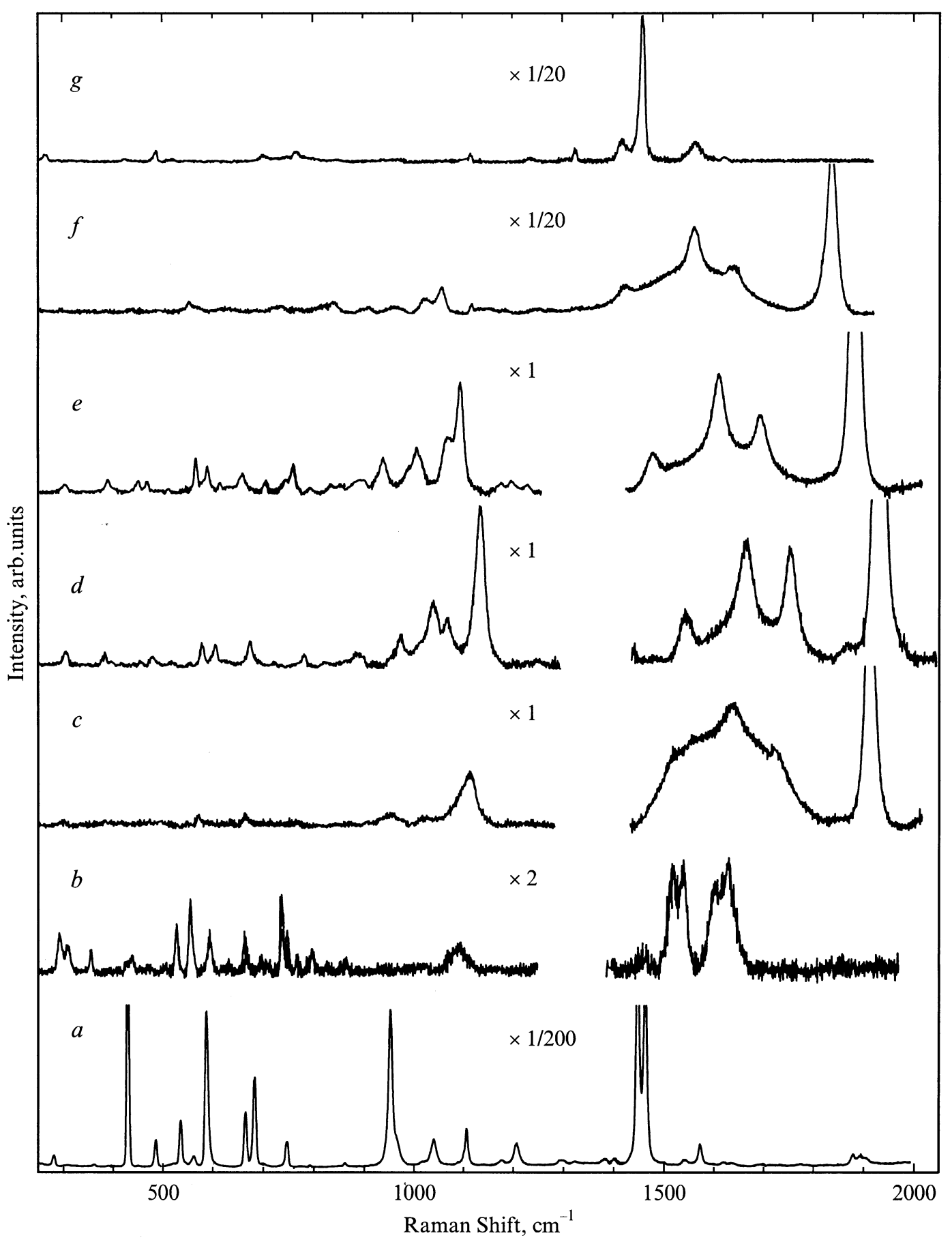

Figure 1. Raman spectra of the tetragonal 2D polymeric phase of $\mathrm{C}_{60}$ at high pressure and room temperature. Spectra $(a),(b),(c),(d)$ are related to the increase of pressure from ambient to $14.1,20.7$, and $27.5 \mathrm{GPa}$, respectively. Spectra $(e)$ and $(f)$ are related to the decrease of pressure from $12 \mathrm{GPa}$ to ambient. Spectrum $(g)$ is related to the principal part of the detonated sample after release of pressure.

peaks can be tracked back to the peaks observed in the tetragonal polymeric phase of $\mathrm{C}_{60}$ and may be related to the fullerene molecular cage. The decrease of pressure (Fig. 1, $e, P \approx 12 \mathrm{GPa}$ ) up to its total release to ambient (Fig. 1, $f$ ) results in the gradual shift of the Raman peaks to lower energies as well as in the preservation of the spectrum to ambient pressure. The pressure dependence of the Raman modes of the tetragonal 2D polymeric phase of $\mathrm{C}_{60}$ in the high-energy region is shown in Fig. 2. The shadowed area marks the pressure region around $\sim 20 \mathrm{GPa}$ where the drastic changes in the Raman spectrum took place. Similar changes, at $\sim 20 \mathrm{GPa}$, are shown also in the pressure dependence of almost all Raman modes. The decrease of pressure from $\sim 27 \mathrm{GPa}$ to ambient, in a time 


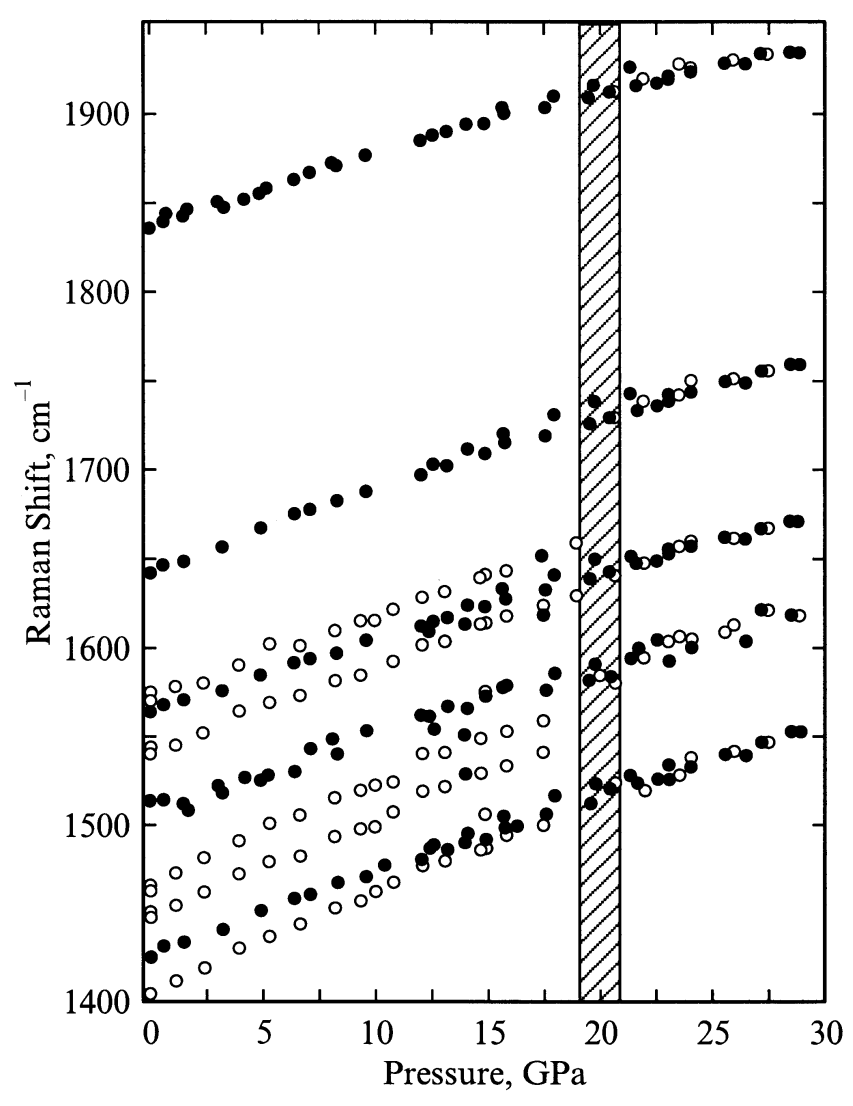

Figure 2. Pressure dependence of the Raman frequencies of the 2D tetragonal polymeric phase of $\mathrm{C}_{60}$ in the high-energy region. Open (closed) symbols are related to the increase (decrease) of pressure. The shadowed area near $20 \mathrm{GPa}$ indicates the pressure region of the irreversible transformation of the material. period of about two weeks, does not lead to any observable changes in the Raman intensity distribution and the new high-pressure phase remains stable. It is important to note that the recovered sample was in fact in a metastable phase, which was detonated by the probing laser beam during the Raman measurements after a time period of a few days from the moment of its exposure to air. The main part of the detonated sample is related to the pristine or partially dimerized $\mathrm{C}_{60}$ (Fig. $1, g$ ), but there was also a small part related to "diamond-like graphite" that has strong Raman peaks at 1339 and $1595 \mathrm{~cm}^{-1}$ resembling those of diamond and graphite, respectively.

The Raman spectrum of the rhombohedral 2D polymeric phase of $\mathrm{C}_{60}$, at normal conditions, is shown in Fig. 3. As in the case of the other polymeric phases of $\mathrm{C}_{60}$, the symmetry lowering results in a very rich Raman spectrum, especially in the low energy region [9]. The spectrum differs in many details from that of the tetragonal $2 \mathrm{D}$ polymer of $\mathrm{C}_{60}$. The important difference is related to the frequency of the pentagonal-pinch (PP) mode, which is gradually softened in the polymeric phases of $\mathrm{C}_{60}$ in accordance to the increasing number of the $s p^{3}$-like coordinated carbon atoms per $\mathrm{C}_{60}$ molecule. Thus the frequency of the PP-mode decreases from $1469 \mathrm{~cm}^{-1}$ for pristine $\mathrm{C}_{60}$, to 1465,1450 , and $1408 \mathrm{~cm}^{-1}$ for dimerized $\mathrm{C}_{60}$, tetragonal and rhombohedral polymeric phases, respectively. The pressure dependence of the Raman modes of the rhombohedral polymeric phase of $\mathrm{C}_{60}$, studied up to $10 \mathrm{GPa}$, is shown in the insert of Fig. 3. The behavior of the material is reversible with pressure (open and closed symbols for increase and decrease of pressure, respectively) and does not show any peculiarity in the pressure range investigated.

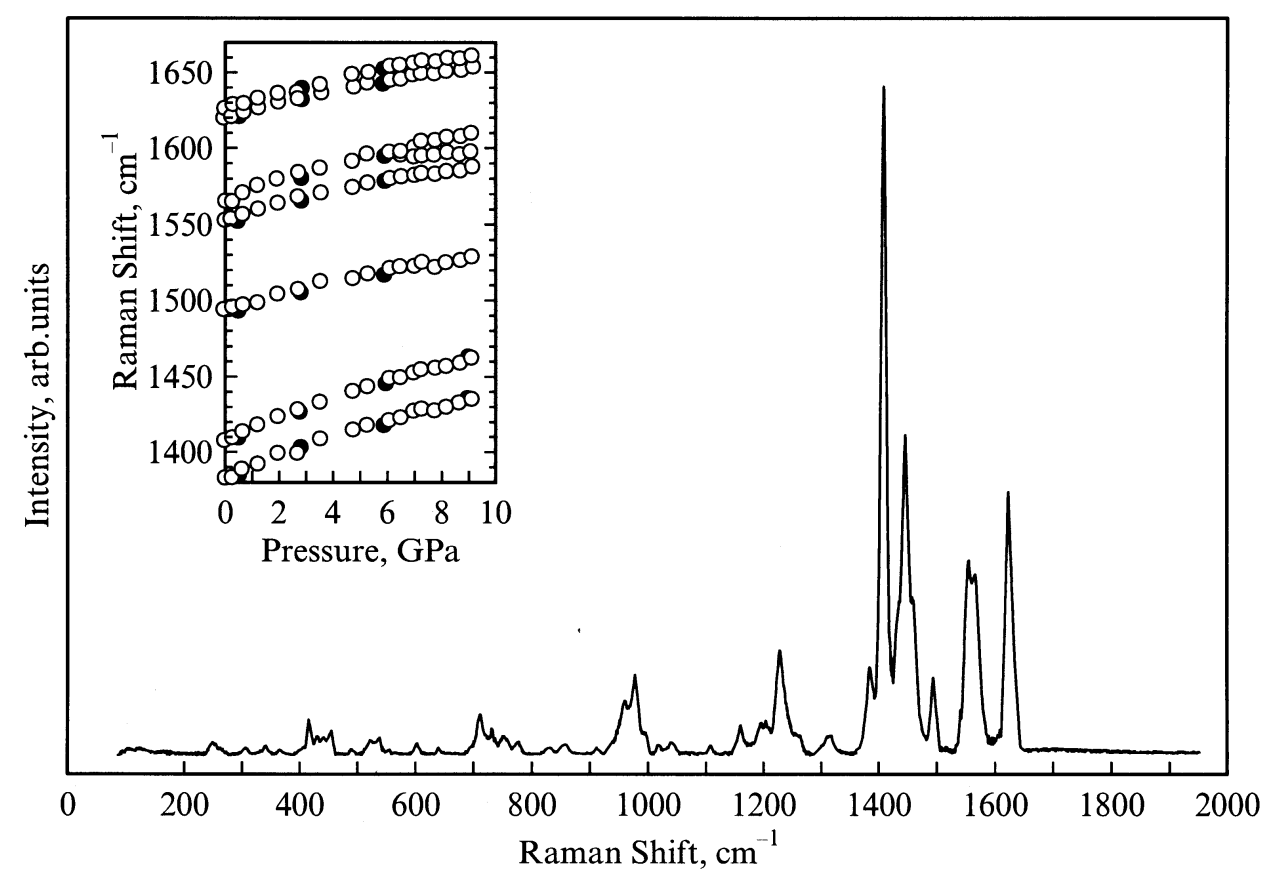

Figure 3. Raman spectrum of the rhombohedral $2 \mathrm{D}$ polymeric phase of $\mathrm{C}_{60}$ at normal conditions. Insert - pressure dependence of Raman frequencies, open (closed) symbols are related to increase (decrease) of pressure. 
The obtained experimental data provide a strong indication that the tatragonal $2 \mathrm{D}$ polymeric phase of $\mathrm{C}_{60}$ undergoes a phase transition at $\sim 20 \mathrm{GPa}$. The transformation is irreversible and takes place via an intermediate disordered pretransitional state. The retention of the phonon modes of the $\mathrm{C}_{60}$ cage and the reduction of the total number of the Raman active modes in the high-pressure phase are indications that this phase may be related to a $3 \mathrm{D}$ polymerised phase. This agrees weel with expected higher symmetry for the 3D polymeric phase. Another argument in favour of the $3 \mathrm{D}$ polymerisation is the matastability of the highpressure phase, which is transformed after a time period into the pristine or partially dimerized $\mathrm{C}_{60}$. The most intriguing feature of the new phase in its Raman spectrum is the presence of the very intense peak at $\sim 1840 \mathrm{~cm}^{-1}$, which cannot be related to any of the fullerene molecular cage modes. The appearance of this peak may be associated to the considerable distortion of the PP-mode of the pristine $\mathrm{C}_{60}$ molecule due to the breakdown of a large number of double $\mathrm{C}=\mathrm{C}$ bonds and the $s p^{3}$-like hybridization of the carbon atoms involved in the intermolecular covalent bonding. A similar string Raman peak near $1840 \mathrm{~cm}^{-1}$, in some chemical compounds of carbon, is related to the stretching vibration of the isolated double $>\mathrm{C}=\mathrm{C}<$ bond [10]. The existence of the $1840-\mathrm{cm}^{-1}$ mode in the high-pressure phase is note compatible with the theoretical calculations in Ref. [7], as they predict no phonon mode above $1600 \mathrm{~cm}^{-1}$. On the other hand the observed transition pressure near $\sim 20 \mathrm{GPa}$ agrees well with the theoretical predictions in [6], therefore, our results point to a polymerization mechanism predicted in this study.

In conclusion, the pressure-induced irreversible transformation of the $2 \mathrm{D}$ tetragonal polymeric phase of $\mathrm{C}_{60}$ has been observed at $\sim 20 \mathrm{GPa}$ by means of in-situ Raman scattering study. The data obtained provide strong indication that the new phase is related to a $3 \mathrm{D}$ polymeric phase of $\mathrm{C}_{60}$. The Raman sudy of the rhombohedral polymeric phase of $\mathrm{C}_{60}$ at pressure up to $10 \mathrm{GPa}$ does not show any structural phase transition or irreversible transformation of the material.

The authors thank Mrs. I. Tsilika for assistance, Profs. Y. Iwasa, K. Prassides and B. Sundqvist for providing of 2D polymeric samples of $\mathrm{C}_{60}$.

\section{References}

[1] A.M. Rao, P. Zhou, K.-A. Wans, G.I. Hager, J.M. Holden, Y. Wang, W.-I. Lee, X.-X. Bi, P.C. Eklund, D.S. Cornett, M.A. Duncan, I.J. Amster. Science 259, 955 (1993).

[2] Y. Ivasa, T. Arimo, R.M. Fleming, T. Siegrist, O. Zhou, R.C. Kaddon, L.J. Rothberg, K.B. Lyons, H.L. Carter Jr., A.F. Hebard, R. Tycko, G. Dalbagh, J.J. Krajevski, G.A. Thomas, T. Yagi. Science 264, 1570 (1994).

[3] M. Nunez-Regueiro, L. Marques, J.-L. Hodeau, O. Bertoux, M. Perraux. Phys. Rev. Lett. 74, 2, 278 (1995).

[4] V.V. Brazhkin, A.G. Lyapin, S.V. Popova. JETP Lett. 64, 11, 802 (1996).
[5] L. Marques, M. Mezouar, J.-L. Hodeau, M. Ninez-Regueiro, N.R. Serebryanaya, V.A. Ivdenko, V.D. Blank, G.A. Dubits. Science 283, 1720 (1999).

[6] S. Okada, S. Saito, A. Oshiyama. Phys. Rev. Lett. 83, 10, 1 986 (1999).

[7] E. Burgos, E. Halac, Ruben Weht, H. Bonadeo, Emilio Artacho, Pablo Ordejon. Phys. Rev. Lett. 85, 11, 2328 (2000).

[8] K.P. Meletov, J. Arvanitidis, I. Tsilika, S. Assimopoulos, G.A. Kourouklis, S. Ves, A. Soldatov, K. Prassides. Phys. Rev. B63, 5, 54106 (2001).

[9] J. Arvanitidis, K.P. Meletov, K. Papagelis, A. Soldatov, K. Prassides, G.A. Kourouklis, S. Ves. Phys. Sat. Sol. (b) 215, 1-2, 443 (1999).

[10] D.A. Long. Raman Spectroscopy. McGraw Hill International Book Company, London (1978). P. 152. 